\title{
A discrete damage zone model for mixed-mode delamination of composites under high-cycle fatigue
}

\author{
Jimenez, Stephen, stephen.k.jimenez@vanderbilt.edu, Vanderbilt University
}

\begin{abstract}
A discrete damage zone model (DDZM) is developed within the finite element framework to simulate mode-mix ratio- and temperature-dependent delamination in laminated composite materials undergoing high cycle fatigue loading. In the DDZM, discrete spring elements are placed at the finite element nodes along the laminate interface. Static and fatigue damage laws are used to define the behavior of the spring elements and model irreversible damage growth. The static damage model parameters are obtained from known material properties and fracture mechanics principles. The fatigue damage model parameters are obtained by calibrating the model to fit published experimental data, and the variation of fatigue parameters with mode-mix ratio is given by a quadratic relation. The DDZM predicts crack growth rates that are in agreement with those given from published literature in which a quadratic relation is used to obtain Paris law parameters for different mode-mix ratios, thus validating the approach. Temperature dependance is implemented using an Arrhenius relation for fatigue damage model parameters, and the critical fracture energy varies with temperature as well. Although the model captures the temperature effects on delamination for mode I and $50 \%$ mode II, the prediction deviates from experiments for pure mode II, because the corresponding damage mechanism entirely changes with temperature. The DDZM converges upon mesh refinement, given that the mesh size used for calibrating model parameters is sufficiently small. The mechanisms driving static and fatigue damage for different static model parameters (i.e., initial stiffness and critical separation) and their influence on overall damage growth are also investigated. It was found that for a low initial spring stiffness fatigue damage dominates the total damage growth, whereas for a large initial stiffness static damage dominates. For intermediate initial stiffnesses, the growth of static and fatigue damage becomes sensitive to mesh size, and model convergence is difficult to attain.
\end{abstract}

